\title{
Quantum scars and partial breaking of ergodicity
}

\section{Probing many-body dynamics on a 51-atom quantum simulator}

Authors: Hannes Bernien, Sylvain Schwartz, Alexander Keesling, Harry Levin,

Ahmed Omran, Hannes Pichler, Soonwon Choi, Alexander S. Zibrov, Manuel Endres, Markus Greiner, Vladan Vuletic and Mikhail D. Lukin

arXiv:1707.04344, Nature 551, 579-584 (2017)

\section{Weak ergodicity breaking from quantum many body scars}

Authors: C.J. Turner, A.A. Michailidis, D.A. Abanin, M. Serbyn and Z. Papic

arXiv:1711.03528, Nature Physics 14, 745-749 (2018)

\section{Recommended with a Commentary by Rahul Nandkishore, University of Colorado at Boulder}

If we have a well isolated many body quantum system which is time evolving according to its own Hamiltonian, then how should we understand the long time limit of the dynamics? Traditional textbooks on quantum statistical mechanics assume that the system goes to thermal equilibrium at long times, at a temperature set by the energy density of the initial condition. However, at least two exceptions to this paradigm are well known. One exception is integrable systems, which have an extensive number of explicit conservation laws. Another is many body localized systems, which have an extensive number of emergent conservation laws. Both integrable and many body localized systems can evade ergodicity, however for integrable systems it remains unknown whether ergodicity breaking is robust to small perturbations of the Hamiltonian, whereas many body localization has only been proven to exist for strongly disordered spin chains [1]. Do there exist alternative routes to ergodicity breaking, which do not rely on the existence of an extensive number of (explicit or emergent) conservation laws, or on strong disorder?

Examples of ergodicity breaking Hamiltonians which are neither integrable nor many body localized have been known in the theory literature for some years. For example, [2] identified a tower of exact non-thermal eigenstates embedded in the spectrum of the (nonintegrable, disorder free) spin-1 AKLT Hamiltonian. Meanwhile, Shiraishi and Mori [3] proposed a generic method by which non-thermal eigenstates could be 'embedded' into the spectrum of an otherwise thermalizing Hamiltonian. The search for new mechanisms for breaking ergodicity really exploded in interest with the publication of the paper by Bernien et al, which provided an experimental example of a system that apparently broke ergodicity, without making use of either integrability or many body localization. 

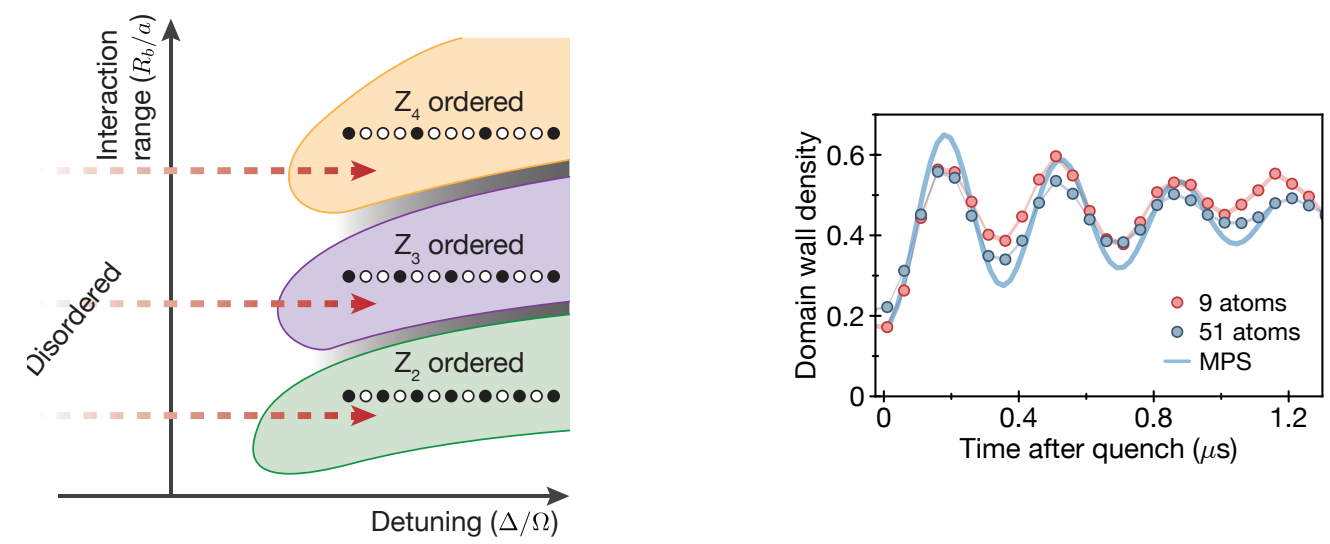

Figure 1: The experiment of Bernien et al realizes a 51 qubit system with a parameter space that contains regimes where the ground state is a charge density wave (left panel). The system is initialized in a period two charge density wave, and then quenched to a parameter regime where this state is at high energy density. The thermalization of such an initial condition would proceed through the proliferation of domain walls in the charge density wave order. However, experimental monitoring of the dynamics (right panel) indicates that the domain wall density is an oscillatory function of time, corresponding to a series of (damped) revivals of the initial condition. These revivals are indicative of an approximate ergodicity breaking in this system. Figures are from arXiv:1707.04344, Nature 551, 579-584 (2017)

The work by Bernien et al experimentally realized a system of 51 qubits based on Rydberg atoms. These Rydberg qubits can be viewed as two level systems - but with a constraint. No two adjacent qubits can be simultaneously in the excited state. The effective Hamiltonian for the system takes the form $H=\sum_{i} P_{i} X_{i+1} P_{i+2}$, where $X_{i}$ is the Pauli $X$ operator acting on site $i$, and $P_{i}=\frac{1}{2}\left(1+Z_{i}\right)$ is the projector onto the 'unexcited' state. We will henceforth refer to this as the PXP Hamiltonian. It can be confirmed, both experimentally and especially numerically, that 'generic' initial states appear to thermalize under the action of the PXP Hamiltonian. However, Bernien et al explored the dynamics starting from an initial condition where every other site was excited (period 2 density wave). This corresponds to an initial condition that is at 'infinite temperature' within the constrained Hilbert space acted on by the initial Hamiltonian. While the density wave order originally decays it then revives periodically, and these revivals persist to times much longer than the thermalization time for generic initial states. Thus, the dynamics starting from this specific initial condition appears non-ergodic - but does not fall within the paradigms of either many body localization or integrability. What is going on?

A possible answer was advanced by Turner et al. They explored the spectrum of the PXP Hamiltonian numerically and observed that while most of the spectrum appeared thermal, there was a 'band' of non-thermal, low entanglement states embedded within the spectrum, and these states had a high overlap with the charge density wave initial condition leading to periodic revivals in the Bernien et al experiment. These special states were dubbed 'many body scars' in analogy with the single particle scar states associated with periodic orbits in otherwise chaotic single particle systems [4]. This catalyzed an explosion of theoretical 
interest in the PXP Hamiltonian. For example, [5] pointed out that the PXP Hamiltonian was 'close to integrable,' while [6] made explicit the connection between the many body scar states of Turner et al and the Shiraishi-Mori formalism [3], [7] made explicit the connection to periodic orbits, and [8] analytically identified exact non-thermal eigenstates embedded within the spectrum. These are just some of the early contributions to what is by now a large literature studying the PXP model and the peculiar ergodicity breaking that arises therein - I have not attempted to provide a comprehensive review, suffice to say the dynamics of this (and related) models is a rich subject under rapid development.

Is the PXP Hamiltonian unique, or is there a more general understanding of such "partially non-ergodic' systems? Moreover, most of the existing literature on scars focuses on specific Hamiltonians (like the PXP Hamiltonian). Does the essential phenomenology survive small perturbations in the thermodynamic limit? This would appear to be a necessary condition for a true 'phase of matter.' It seems worth mentioning in this context the recent demonstration $[9,10]$ that just two local conservation laws can be sufficient to ensure the appearance of a subspace exponentially large in system volume, within which the dynamics is provably localized, even while the rest of the spectrum may be thermalizing, and with the localized subspace provably robust to arbitrary local perturbations respecting the two conservation laws. This result extends to systems in $d$ spatial dimensions with $d+1$ conservation laws [11], and provides a proof of principle that partially non-ergodic 'phases' can exist. What a complete theory of 'partially non-ergodic' systems looks like remains an open question, but one under intense investigation.

\section{References}

[1] J. Z. Imbrie, Jour. Stat. Phys. 163:998-1048 (2016)

[2] S. Moudgalya, S. Rachel, B. A. Bernevig and N. Regnault, Phys. Rev. B 98, 235155 (2018)

[3] N. Shiraishi and T. Mori, Phys. Rev. Lett. 119030601 (2017)

[4] E. J. Heller, Phys. Rev. Lett. 53, 1515-1518 (1984)

[5] V. Khemani, C. Laumann and A. Chandran, Phys. Rev. B 99, 161101(R) (2019)

[6] S. Choi, C.J. Turner, H. Pichler, W.W. Ho, A.A. Michailidis, Z. Papic, M. Serbyn, M.D. Lukin and D.A. Abanin, Phys. Rev. Lett. 122, 220603 (2019)

[7] W.W. Ho, S. Choi, H. Pichler and M.D. Lukin, Phys. Rev. Let. 122, 040603 (2019)

[8] C.-J. Lin and O. I. Motrunich, Phys. Rev. Lett. 122, 173401 (2019)

[9] V. Khemani and R. Nandkishore, arXiv: 1904.04815

[10] P. Sala, T. Rakovszky, R. Verresen, M. Knap and F. Pollmann, arXiv: 1904.04266

[11] V. Khemani, M. Hermele and R. Nandkishore, arXiv: 1910.01137 